\title{
Three-Photon Absorption in ZnO Film Using Ultra Short Pulse Laser
}

\author{
Raied K. Jamal, Mohammed T. Hussein, Abdulla M. Suhail \\ Department of Physics, College of Science, University of Baghdad, Baghdad, Iraq \\ Email: raiedkamel@yahoo.com, Abdulla_sh1@yahoo.com,mohammedtaki97@yahoo.com, hani_saka@yahoo.com
}

Received January 4, 2012; revised February 1, 2012; accepted February 27, 2012

\begin{abstract}
The three-photon absorption (3PA) in nanostructure wide-band gap $\mathrm{ZnO}$ semiconductor material is observed under high intensity femtosecond Titanium-Sapphire laser of $800 \mathrm{~nm}$ wavelength excitation. The $\mathrm{ZnO}$ film was prepared by chemical spray pyrolysis technique with substrate temperature of $400^{\circ} \mathrm{C}$. The optical properties concerning the absorption, transmission, reflection, Raman and the photoluminescence spectra are studied for the prepared film. The structure of the $\mathrm{ZnO}$ film was tested with the X-ray diffraction and it was found to be a polycrystalline with recognized peaks oriented in (002), and (102). The measured of three photon absorption coefficient was found to be about 0.0166 $\mathrm{cm}^{3} / \mathrm{Gwatt}^{2}$, which is about five times higher than the bulk value. The fully computerized z-scan system was used to measure the nonlinear coefficients from the Gaussian fit of the transmitted laser incident.
\end{abstract}

Keywords: Multiphoton Processes; ZnO Nanocrystalline; Nonlinear Optics

\section{Introduction}

The $\mathrm{ZnO}$ nanostructures have many applications in gas sensors, UV detectors and solar cells [1-3]. This material has some additional advantages compared to other large band-gap semiconductors; for example, its large exciton binding energy (about $60 \mathrm{meV}$ ) which is three times the binding energies of $\mathrm{ZnSe}$ and $\mathrm{GaN}$ [4]. This allows a stable exciton distribution and achieves efficient excitonic emission at room temperature. The optical and electrical properties of $\mathrm{ZnO}$ nanostructures are studied at different preparation techniques and at different substrate materials $[5,6]$. The pumping of $\mathrm{ZnO}$ crystalline nanofilm with near-infrared femtosecond radiation pulses, enhances the nonlinear interaction between the ultrahigh intensity applied optical field and the $\mathrm{ZnO}$ nanostructures $[7,8]$. The nonlinear interaction leads to the simultaneous absorption of two or more photons of subband gap energy. The absorption is through a virtual-states that assists the inter band transitions. This transition produces electron-hole pairs in the excited states and, subsequently, the band-edge emission via their radiative recombination [9]. The two photon absorption (2PA) in semiconductor nanocrystals $(\mathrm{NCs})$ has been widely investigated $[8,10]$, while the research effort on their three-photon absorption (3PA) is limited [11]. The three photon absorption was observed in $\mathrm{ZnO}$ and $\mathrm{ZnS}$ crystals when pump with lasers of ultra excitation irradiance which was more than $40 \mathrm{GW} / \mathrm{cm}^{2}$ [12-15]. Three-photon absorption in nanos- tructure wide-Band gap semiconductor $\mathrm{ZnO}$ using femtosecond laser for thin film was studied by [16].

In this work the three photon absorption in $\mathrm{ZnO}$ nanostructure illuminated by intensity $110 \mathrm{GW} / \mathrm{cm}^{2}$ Ti-Sapphire laser is observed. The work concentrates on the effect of the nanostructure on the nonlinear parameters through the studying of the 3PA coefficient and the laser threshold pumping power. Simple mathematical relations are developed to describe the dependence of the fluorescent emission on the pumping laser intensity. The three photon absorption coefficient was calculated from the experimental measurements.

\section{Experimental Work}

The $\mathrm{ZnO}$ film was prepared by chemical spray pyrolysis technique. The film was deposited on quartz substrates heated to $400^{\circ} \mathrm{C}$. The spray solution is prepared by mixing Zinc Chloride $\left(\mathrm{ZnCl}_{2}\right)$ at $0.1 \mathrm{M}$ with distillated water. The above mixture solution was placed in the flask of the atomizer and spread by controlled nitrogen gas flow on the heated substrates. The chemical spray pyrolysis experimental setup is similar to the standard unit fully described by [17]. The spraying time was controlled by adjustable a solenoid valve. The heated substrate was left for $12 \mathrm{sec}$ after each spraying run to give time for the deposited $\mathrm{ZnO}$ layer to be dry. In order to get film of proper thickness many layers deposited of $\mathrm{ZnO}$ are required. The optimum experimental conditions for ob- 
taining homogeneous $\mathrm{ZnO}$ film at $400^{\circ} \mathrm{C}$ are determined by the spraying time, the drying time and the flashing gas pressure. The schematic representation of the spray system is given in Figure 1. The possible reaction of the spray chemical on the heated substrate is yielding for the following reaction:

$$
\mathrm{ZnCl}_{2}+\mathrm{H}_{2} \mathrm{O} \underset{400^{\circ} \mathrm{C}}{\stackrel{\text { Heat }}{\longrightarrow}} \mathrm{ZnO}(\text { film/substrate })+2 \mathrm{HCl}
$$

During the chemical reaction, gas and water vapor is obtained from this reaction due to the high temperature of the substrate. At end of reaction a white precipitates remain from the reaction as a nanofilm of $\mathrm{ZnO}$ as shown in Figure 2. The topography study of the prepared nanofilm surface was carried on using Scanning Electron Microscopy (SEM) type ULTRA 55 with different magnification; as shown in Figure 3. The X-ray diffraction (XRD) pattern of $\mathrm{ZnO}$ film was recorded by XDR 2000. The X-ray diffractometer use copper tube radiation line of wavelength $1.54 \AA$ in $2 \theta$ range from $20^{\circ}$ to $60^{\circ}$. The scanning rate was $1 \mathrm{deg} / \mathrm{min}$.

The UV-VIS-NIR absorption, transmission and reflection spectra of the sample were recorded by Hitachi U-4100 spectrometer covering the spectral range 200 $1100 \mathrm{~nm}$. The photoluminescence spectrum (PL) was studied using SL1174 spectrophotometer in the range $300-900 \mathrm{~nm}$.

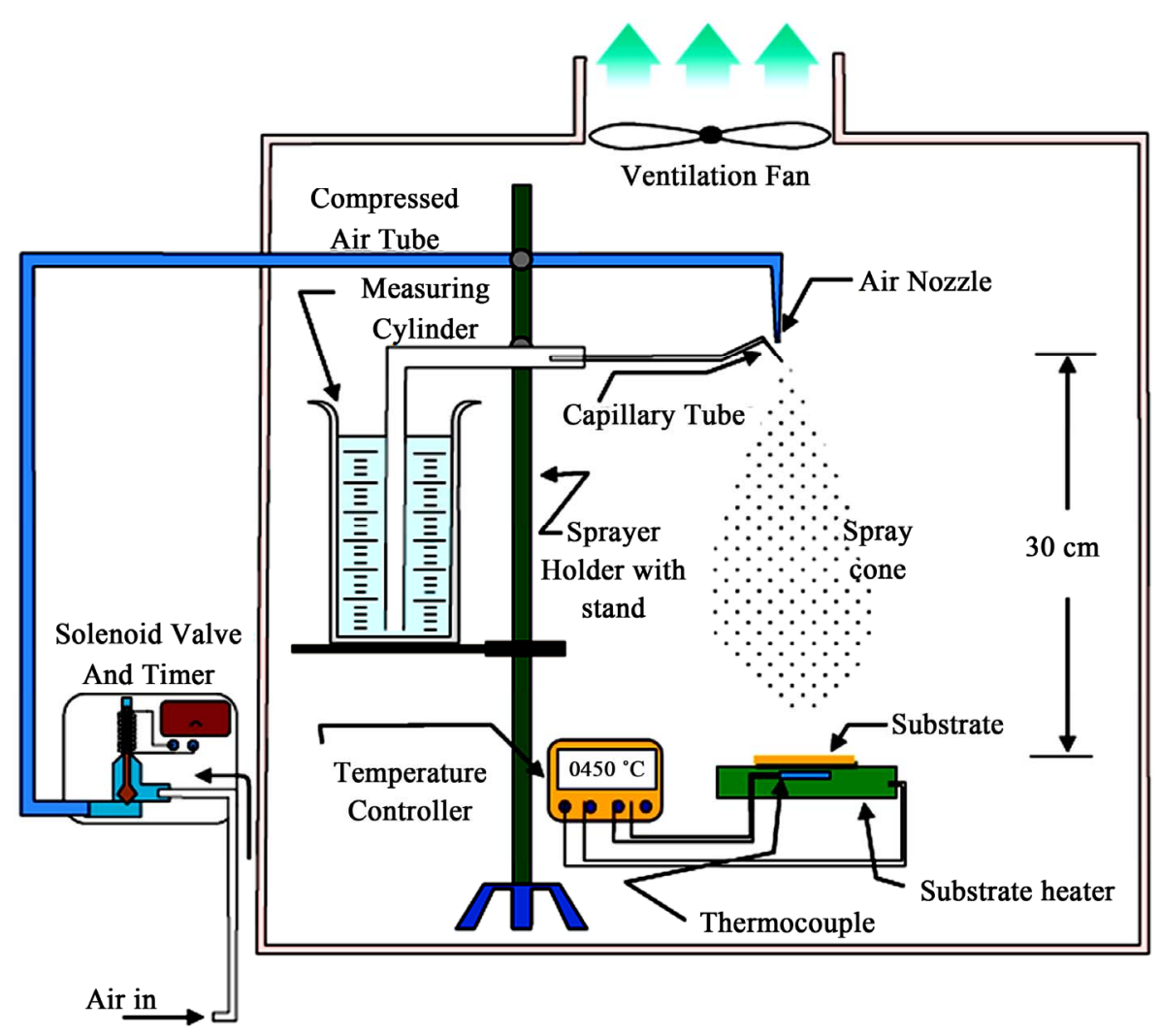

Figure 1. Schematic representation of the spray system.

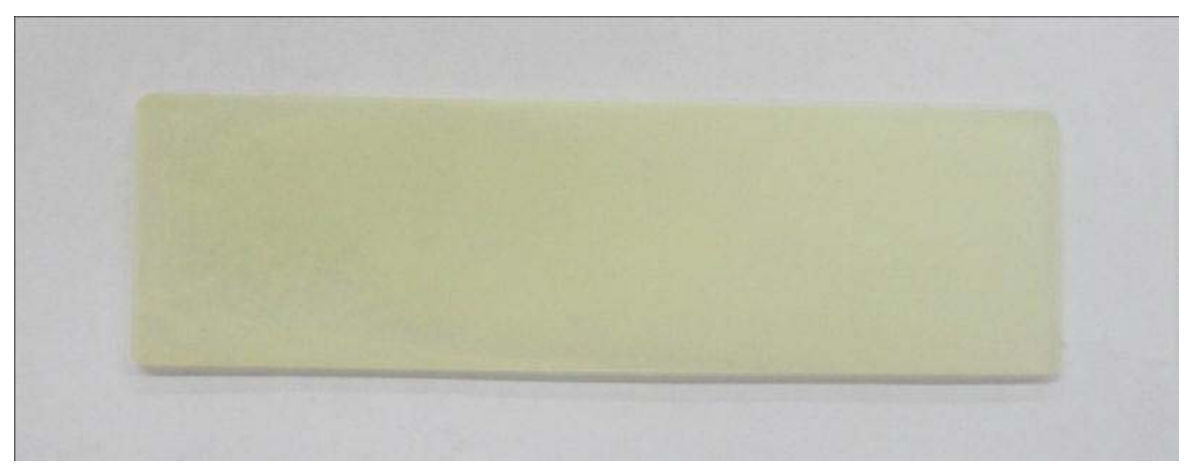

Figure 2. A photo of spray pyrolyzed $\mathrm{ZnO}$ nanofilm on glass samples. 


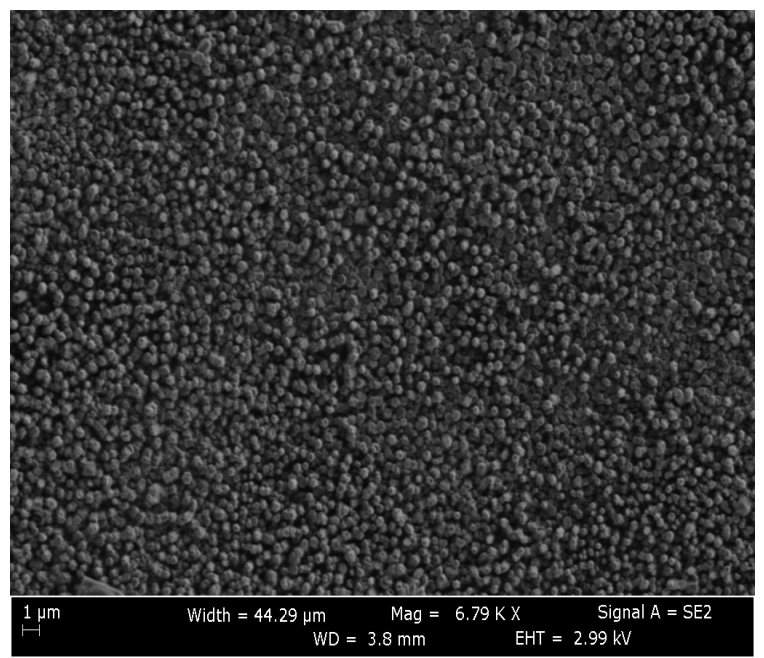

(a)

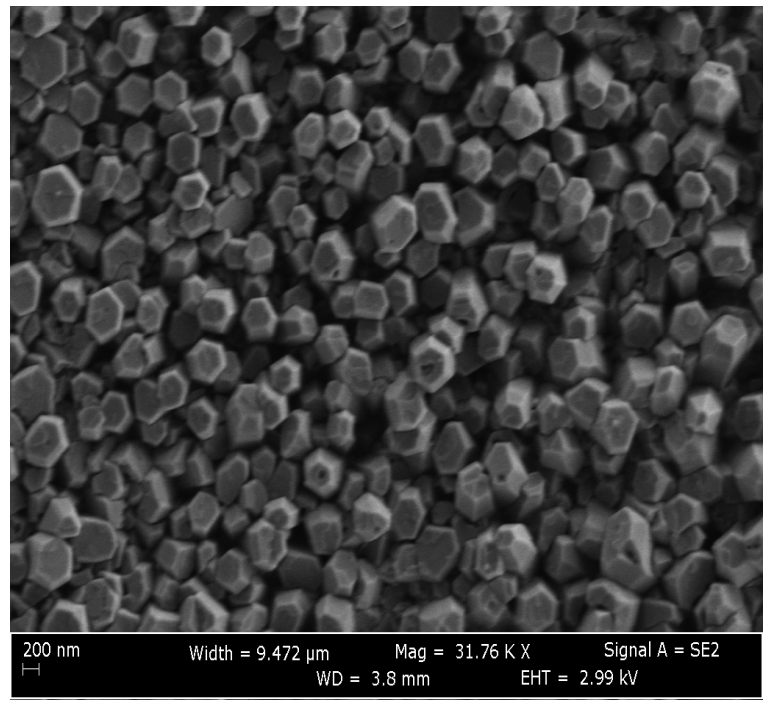

(c)

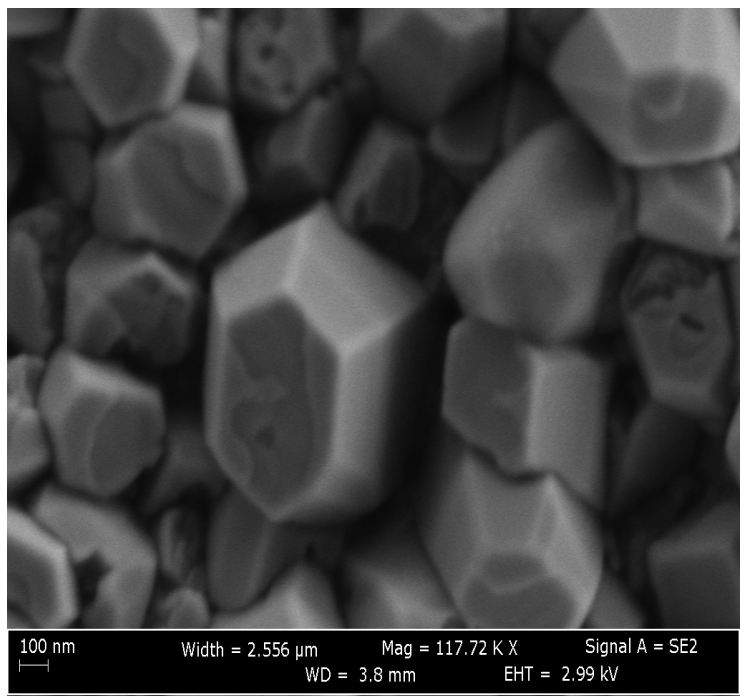

(e)

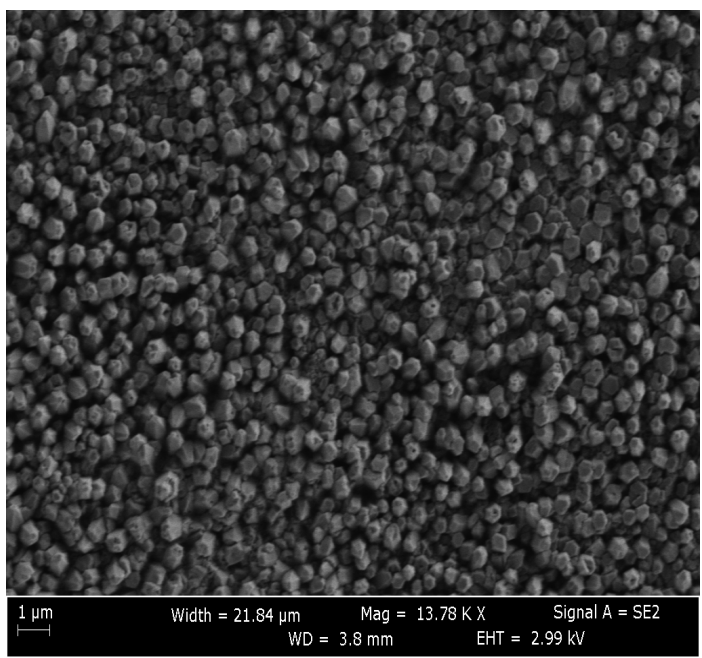

(b)

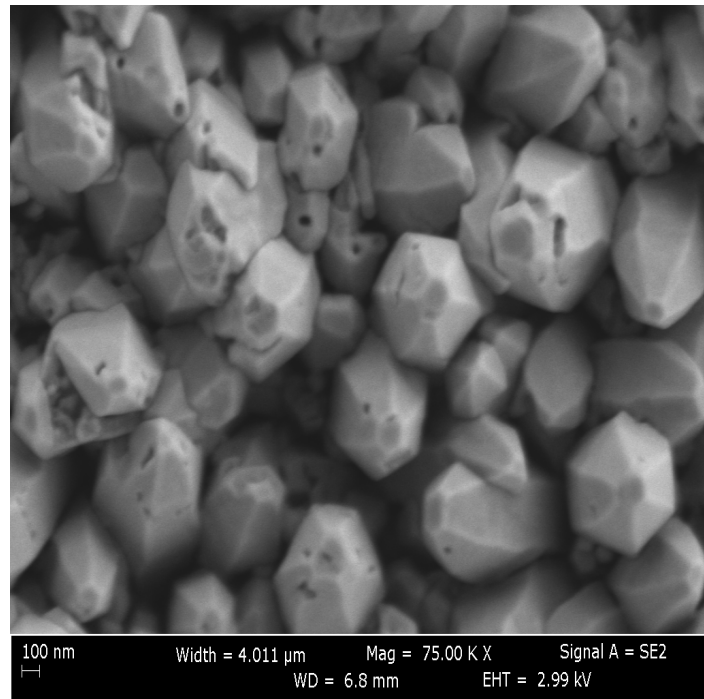

(d)

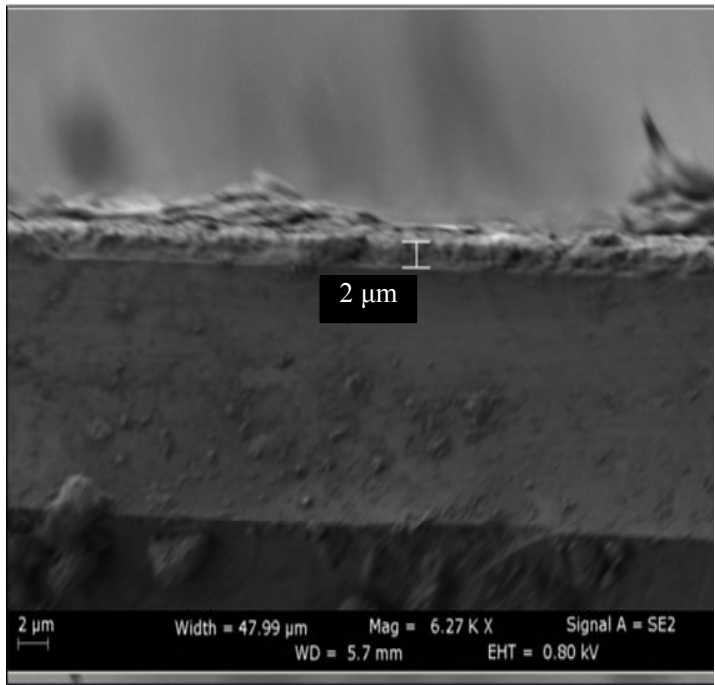

(f)

Figure 3. (a)-(e) represent SEM images of ZnO nanofilm at different magnification power and (f) for a sample thickness. 
The nonlinear absorption study at the near resonant regime was carried out using single beam femtosecond $z$-scan technique. The $z$-scan setting is illustrated by schematic diagram shown in Figure 4. A femtosecond laser of pulse duration $60 \mathrm{fs}$ and of average power 0.165 $\mathrm{W}$ was used as a laser source. The pulse duration was measured by autocorrelation system and the energy was measured by pyroelectric energy prob model type (PDA36A), covering the rang 350 - $1100 \mathrm{~nm}$ from THORLABS. The beam profile was adjusted by spatial filter leading to spatial intensity profile near Gaussian with beam quality of $\mathrm{M}^{2} \approx 1.79$. The laser beam was focused by a lens of $15 \mathrm{~cm}$ focal length to produce a waist of $22.5 \mu \mathrm{m}$. The sample was translated along the beam axis ( $z$-axis) through the Rayleigh distance $2000 \mu \mathrm{m}$. The distance from lens to aperture was $53 \mathrm{~cm}$ and beam diameter at detector was $16.4 \mathrm{~mm}$.

\section{Results and Discussion}

The topography study of the prepared film shows the formation of the $\mathrm{ZnO}$ nanostructure and the film thickness was around $2 \mu \mathrm{m}$. as reveled by Scanning Electron Microscopy (SEM) type ULTRA 55 with different magnification; as shown in Figure 3. These figures showed nanocrystals of size $\sim(100-200 \mathrm{~nm})$. The sample was scanned in all zones before the picture was taken. The micrographs reveled that the particles were hexagonal in shape. This indicated that $\mathrm{ZnO}$ nanocrystal were grown with c-axis orientation and almost perpendicular to the substrate surface as observed in the scanning electron microscope (SEM) image.

The XRD pattern of $\mathrm{ZnO}$ film prepared with $2 \mu \mathrm{m}$ thickness is illustrated in Figure 3. The spectrum indicates that the $\mathrm{ZnO}$ film is a polycrystalline structure. The observed values of the XRD peaks are compared with American Society for testing and Material (ASTM) data for hexagonal zinc oxide. It can be noticed from the XRD pattern the strongest peak observed at Bragg's angle $2 \theta=$ $34.38^{\circ}$ can be attributed to the (002) plan of the hexagonal $\mathrm{ZnO}$ and the interplanar distance can be determined by Bragg's law, $d_{002}=0.26 \mathrm{~nm}$. The (102) peak is also observed at $2 \theta=47.44^{\circ}$ but this peak is much lower intensity than the (002) peak. The figure shows broad peaks which give evidence of the nanostructure formation. Using the width of (002) peak which appears at angle $34.38^{\circ}$ on $2 \theta$ scale in Scherrer's formula [18]:

$$
d=0.94 \lambda / \beta \cos \theta
$$

where $d$ is the average crystalline grain size, $\lambda$ is the wavelength, $\beta$ represents the full width at half maximum (FWHM) in radian and $\theta$ is the Bragg diffraction angle in degree. The size of the formed nanoparticles was found to be about $46 \mathrm{~nm}$, this value is close from the value that measured by SEM. Using the width of (102) peak which appears at angle $47.44^{\circ}$ on $2 \theta$ scale the size of the formed nanoparticles was found to be about $26 \mathrm{~nm}$. The interplaner distance can be determined by Bragg's law $d_{102}=$ $0.19 \mathrm{~nm}$.

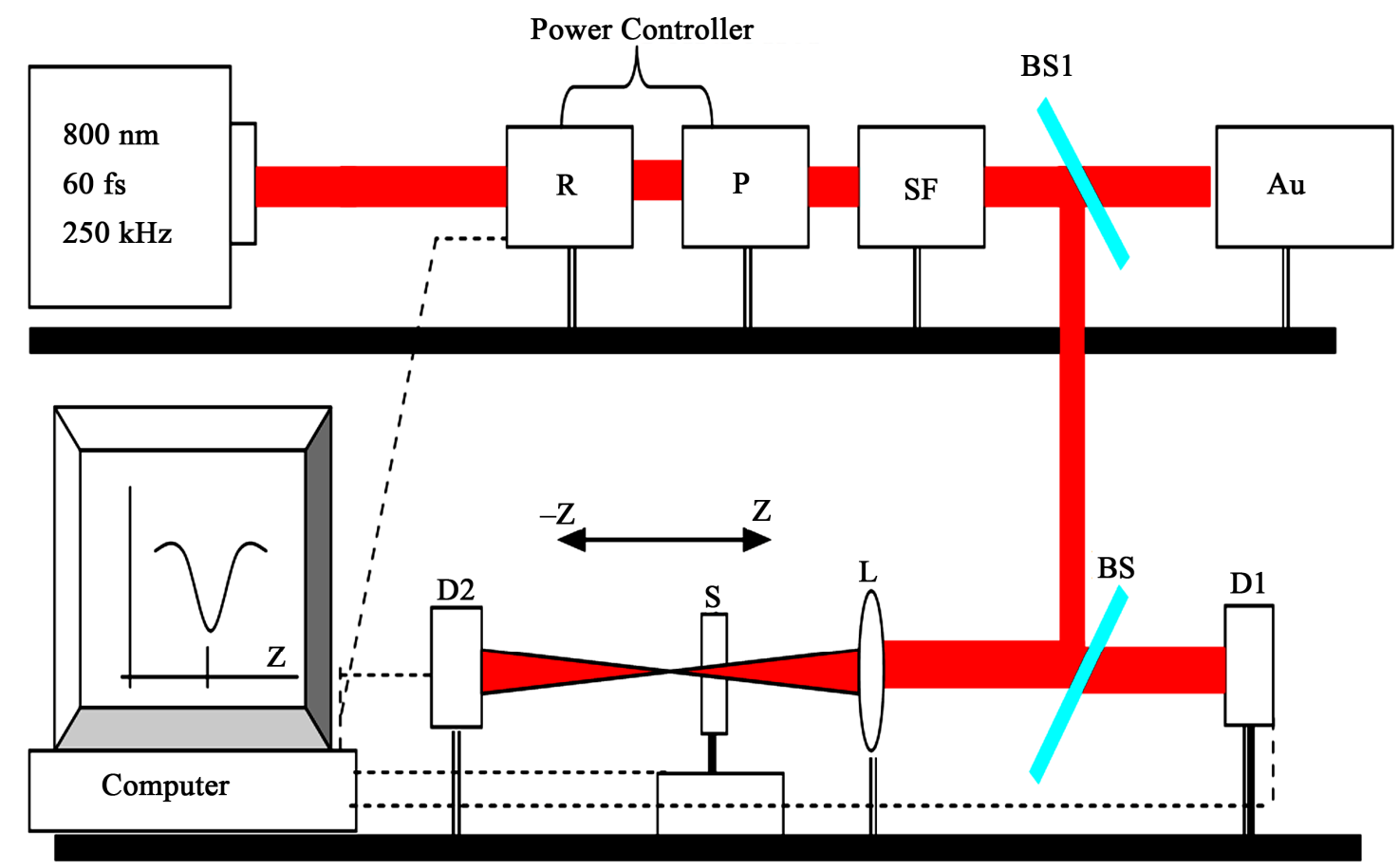

Figure 4. Schematic of the z-scan setup recording the nonlinear absorption, R-rotator, P-polarizer, SF-spatial filter, BS1, BS2-beam splitter, Au-autocorrelation, D1,D2-detectors, L-lens, S-sample. 
The optical properties of $\mathrm{ZnO}$ film which were prepared on quartz substrate have been studied in this work. The absorption spectrum of the $\mathrm{ZnO}$ film is shown in Figure 5. This spectrum shows low absorption in the visible and infrared regions; however, the absorption in the ultraviolet region is high. This result were in agreement with the absorption spectrum which obtained by other workers. The energy band gap of $\mathrm{ZnO}$ film was estimated using Tauc equation which can be written as:

$$
(\alpha h v)=A(h v-E g)^{n}
$$

where $A$ is a constant, $\alpha$ absorption coefficient, $h v$ the photon energy $(E g)$ the band gap, $n=1 / 2$ for the direct transitions. Referring to the data extracted from the absorption spectrum in Figure 6, the absorption coefficient $(\alpha)$ was calculated as a function of wavelength. Assuming allowed transition; the dependence of $(\alpha h v)^{2}$ on $(h v)$ is plotted as in Figure 7. The extrapolation of the linear part of the plot $(\alpha h v)^{2}=0$, gives rise on estimation of the energy gap value of the prepared $\mathrm{ZnO}$ film. The value of the energy gap was found to be about $3.3 \mathrm{eV}$. This value was in a good agreement with values mentioned by other works.

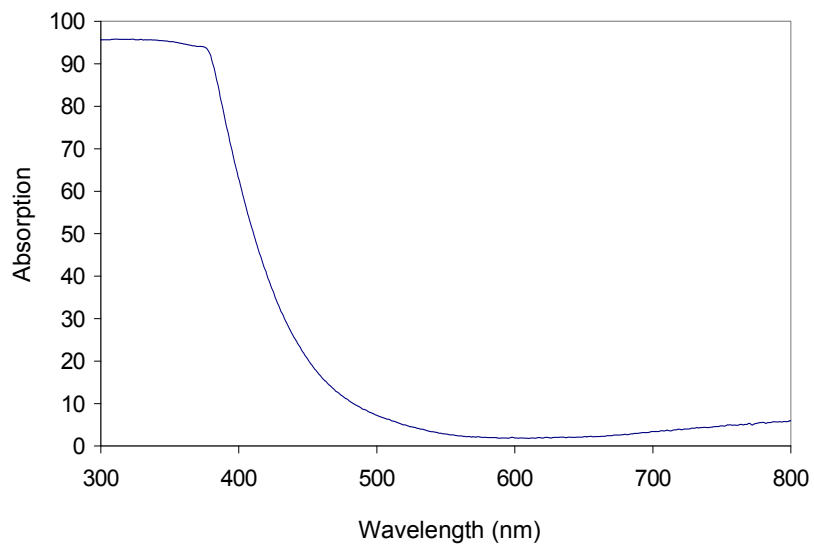

Figure 5. UV-VIS absorption spectra of ZnO nanofilm.

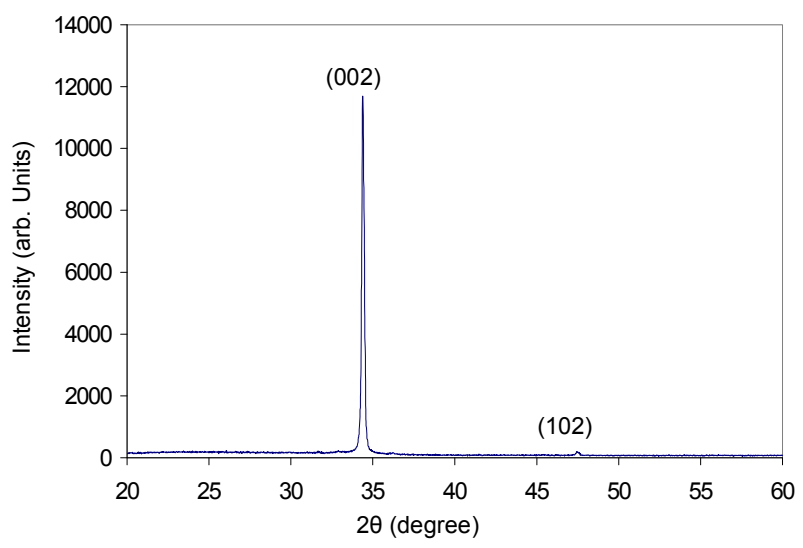

Figure 6. XRD pattern of $\mathrm{ZnO}$ nanofilm deposited on a glass substrate at $400^{\circ} \mathrm{C}$.
The optical transmittance spectrum of the $\mathrm{ZnO}$ film is shown in Figure 8. It can be noticed from this figure that the transmittance is high in the visible and infrared regions and low transmission in ultraviolet region.

The optical reflection spectrum of the $\mathrm{ZnO}$ film is shown in Figure 9. It can be noticed from this figure that the maximum reflection at $470 \mathrm{~nm}$ and minimum value was $390 \mathrm{~nm}$. The optical fluorescence spectrum of the

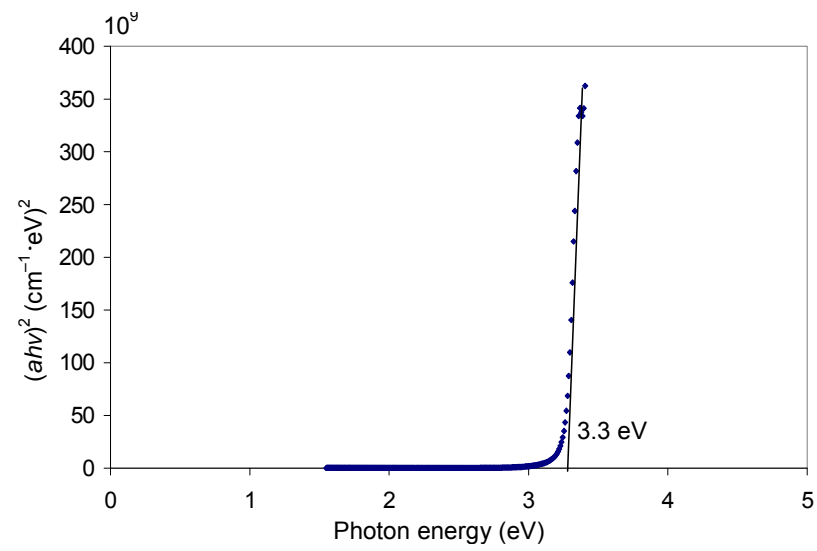

Figure 7. Plot of $(a h v)^{2}$ versus photon energy $h v$ for $\mathrm{ZnO}$ nanonanofilm.

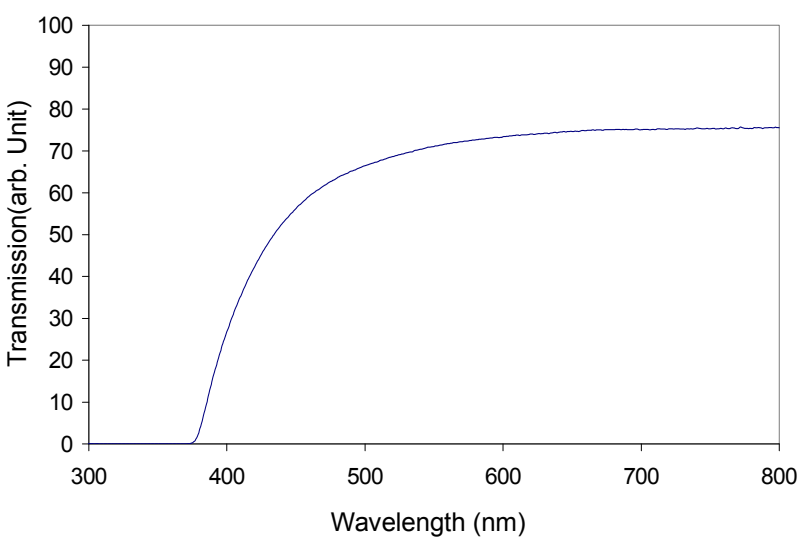

Figure 8. Optical transmission spectra of $\mathrm{ZnO}$ nanofilm.

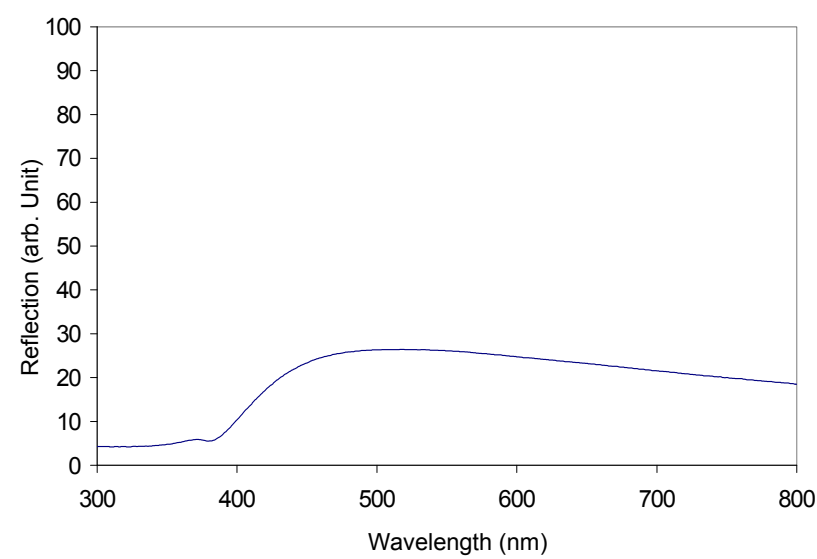

Figure 9. Optical reflection spectra of $\mathrm{ZnO}$ nanofilm. 
$\mathrm{ZnO}$ film illuminated by $320 \mathrm{~nm}$ UV line is shown in Figure 10. The spectrum displays two major luminance peaks at $380 \mathrm{~nm}$ and around $556 \mathrm{~nm}$. The first peak is due to the energy gap transmission which corresponds to $3.28 \mathrm{eV}$. The second peak is due to the excitonic emission; and it is in a good agreement with the results measured by many other authors $[19,20]$. The broad green emission peak that is dominated at $556 \mathrm{~nm}(\approx 2.23 \mathrm{eV})$ is a good evidence for the exciton formation in $\mathrm{ZnO}$ with a binding energy of $60 \mathrm{meV}$. The high binding energy enables the finding of the exciton at room temperature. The intensity at the $556 \mathrm{~nm}$ peak is higher than that found around $380 \mathrm{~nm}$ peak. This is because the band-to-band transition was quenched by the defect states. The same behavior was observed by [21].

The Raman active zone-center optical phonons predicated by the group theory are $A_{1}+2 E_{2}+E_{1}$. The phonons of $A_{1}$ and $E_{1}$ symmetry are polar phonons and, hence, exhibit different frequencies for the transverse- optical (TO) and longitudinal optical (LO) phonons. In wurtzite $\mathrm{ZnO}$ crystals, The non-polar phonon modes with symmetry $E_{2}$ have two frequencies, $E_{2}($ high) is associated with oxygen atoms and $\mathrm{E}_{2}$ (low) is associated with $\mathrm{Zn}$ sublattice. The described phonon modes have been reported in the Raman scattering spectra of bulk $\mathrm{ZnO}[22,23]$. In Figure 11, we present a typical non-resonant Raman scattering spectrum from $\mathrm{ZnO}$ nanocrystal obtain under the $633 \mathrm{~nm}$ non-resonant excitation on of He-Ne laser. For comparison, the phonons peaks observed in bulk $\mathrm{ZnO}$ crystal are summarized in Table $\mathbf{1}$.

In our experiment, Raman spectra were recorded at room temperature under the $633 \mathrm{~nm}$ excitation and laser power $75 \mathrm{~mW}$, the $\mathrm{E}_{2}$ (low) peak is red shifted by $\sim 3 \mathrm{~cm}^{-1}$ from its bulk value, $E_{2}$ (high) peak is red shift by $\sim 2 \mathrm{~cm}^{-1}$, but the peak $\mathrm{E}_{1}(\mathrm{LO})$ at $591 \mathrm{~cm}^{-1}$ corresponding to $\mathrm{E}_{1}(\mathrm{LO})$. The $A_{1}(\mathrm{TO}), \mathrm{E}_{1}(\mathrm{TO}), \mathrm{A}_{1}(\mathrm{LO})$ peaks being absent in this sample.

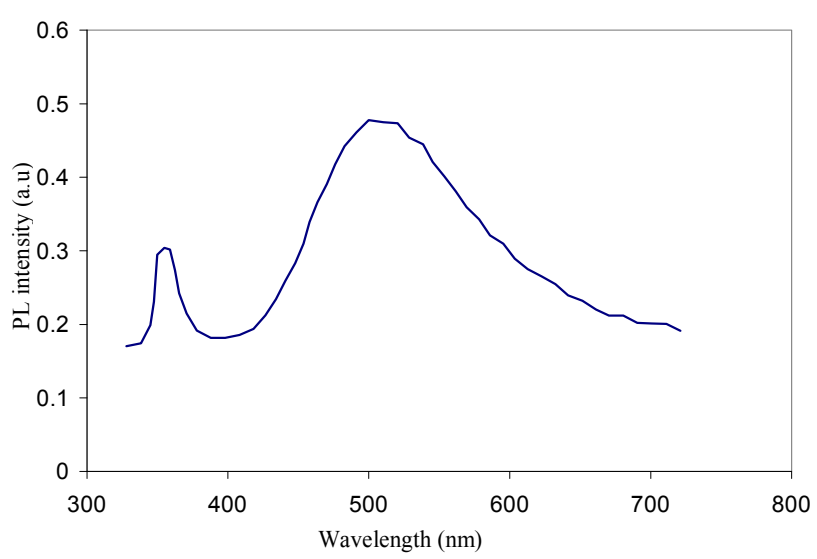

Figure 10. Photoluminescence emission spectrum of $\mathrm{ZnO}$ nanofilm.
There are three possible mechanisms for the phonon peak shift in Raman spectra of nanostructures. The first one is spatial confinement within the quantum dot (nanocrystal) boundaries. The second one is related to the phonon localization by defect. Nanocrystal or quantum dots, produced by chemical methods or by the molecularbeam epitaxy, normally have more defect than corresponding bulk crystal. In order elucidate the possible of the peak shifts we carried out simple calculations in the framework of the H. Richer [24] and I. H. Chambell [25] phenomenological models.

The $\mathrm{z}$-scan transition curve at $110 \mathrm{GW} / \mathrm{cm}^{2}$ excitation intensity is recorded for the nanoparticles $\mathrm{ZnO}$ film and it shown in Figure 12, Where the average power was 0.165 $\mathrm{W}$, Repetition rate (RR) was $250 \mathrm{kHz}$, Pulse energy $E_{\mathrm{p}}$ was $6.6 \times 10^{-7} \mathrm{~J}$, Pulse duration (FWHM) was $60 \mathrm{fs}$, Peak power (PP) was $10.34 \times 10^{6} \mathrm{~W}$.

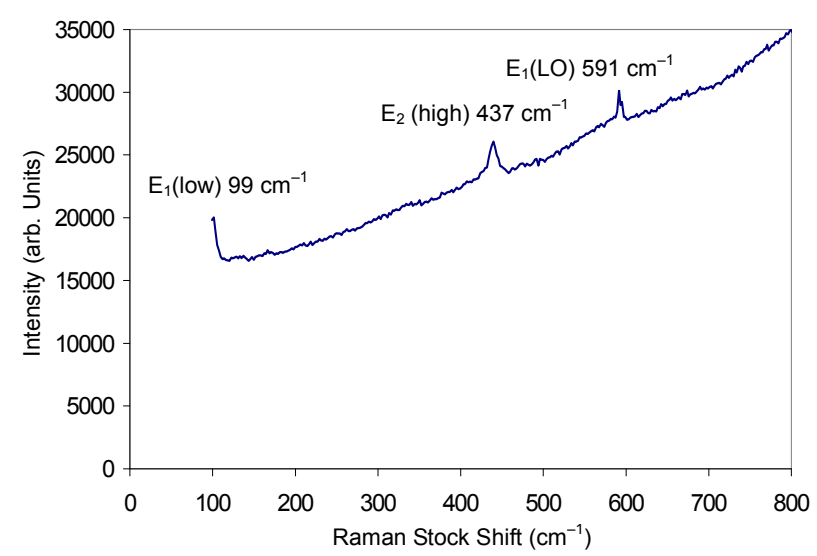

Figure 11. Non-resonant Raman spectra from ZnO nanocrystal under $633 \mathrm{~nm}$ excitation and $75 \mathrm{~mW}$ for He-Ne laser.

Table 1. Raman active phonon mode frequencies (in $\mathbf{c m}^{-1}$ ) for bulk ZnO.

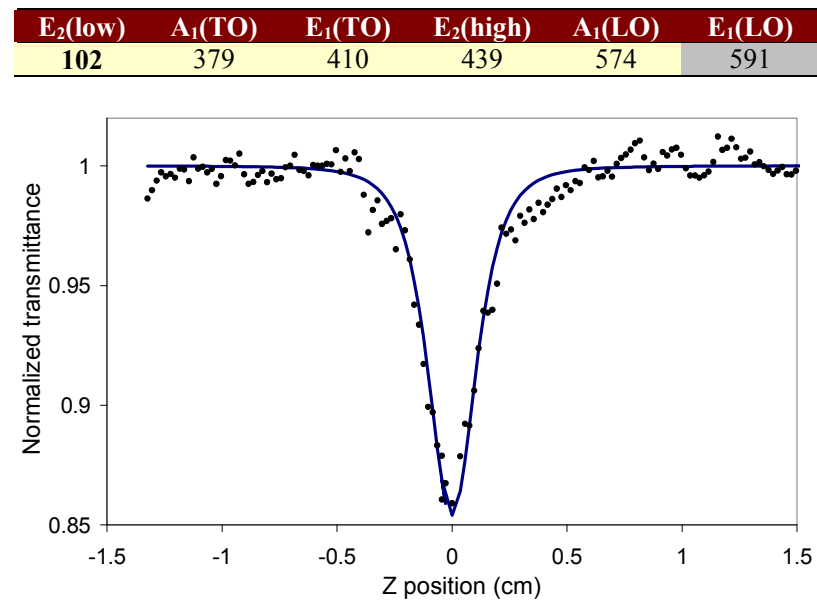

Figure 12. OA Z-scan measured at irradiance $110 \mathrm{GW} / \mathrm{cm}^{2}$, wavelength $800 \mathrm{~nm}$, a pulse duration 60 fs and repetition rate of $250 \mathrm{kHz}$. The solid line is the fitting curve employing the z-scan theory, described in the text, on 3PA. 
The rate equations model describing the transitions between the valance band and the conduction band in our semiconductor sample illuminated by Titanium-Sapphire femtosecond laser of $800 \mathrm{~nm}$ wavelength can be written as:

$$
\begin{aligned}
\frac{\mathrm{d} N_{h}}{\mathrm{~d} t} & =-\frac{\alpha I^{3}}{3 \hbar \omega}+\frac{N_{e}}{\tau_{i}} \\
\frac{\mathrm{d} N_{e}}{\mathrm{~d} t} & =\frac{\alpha I^{3}}{3 \hbar \omega}-\frac{N_{e}}{\tau_{i}}
\end{aligned}
$$

where $N_{h}$ and $N_{e}$ are the population in the valance band and the conduction band respectively. $\alpha$ is the three photon absorption coefficient, $\hbar \omega$ is the energy of the IR pump Titanium-Sapphire laser photon, $I$ is the laser intensity at the sample position and $\tau_{i}$ is the life time of the excited level in the conduction band. The Gaussian laser beam intensity is given by:

$$
I=I_{o}\left[\frac{\omega_{o}^{2}}{\omega^{2}(z)}\right] \exp \left[-\frac{2 r^{2}}{\omega^{2}}\right] \exp \left[-\frac{t^{2}}{\tau_{p}^{2}}\right]
$$

where $I_{o}$ is the laser intensity at the waist of the beam and $\tau_{p}$ is the laser pulse duration. Since the measuring intensity was carried out at the focal point, in the Z-coordinate, thus the intensity in Equation (4) can be reduced to $I=I_{o}$. In order to find the relation between the power of the out put emitted fluorescent pulse and the intensity of the Titanium-Sapphire laser pump, Equation (5) can be arranged as:

$$
\frac{\mathrm{d} N_{e}}{\mathrm{~d} t}+\frac{N_{e}}{\tau_{i}}=\frac{\alpha I_{o}^{3}}{3 \hbar \omega}
$$

The solution of Equation (7) can be written as:

$$
N_{e}=\frac{\alpha \tau_{i} I_{o}^{3}}{3 \hbar \omega}\left[1-e^{-\frac{\tau_{i}}{\tau_{p}}}\right]
$$

Since the pulse duration of the pump laser $\left(\tau_{p}\right)$ is in femtosecond and the life time of the excited state $\left(\tau_{i}\right)$ in picosecond [26], the Equation (8) can be simplified as:

$$
N_{e}=\frac{\alpha \tau_{p}}{3 \hbar \omega} I_{o}^{3}
$$

Multiply both sides of the above equation by the volume $(V)$ of the illuminated part of the $\mathrm{ZnO}$ sample and arranged the terms, Equation (9) can be written as:

$$
\frac{3 \hbar \omega \cdot N_{e} \cdot V}{\tau_{p}}=P_{f}=\alpha \cdot V \cdot I_{o}^{3}
$$

where $P_{f}$ is the power of the emitted fluorescent from the $\mathrm{ZnO}$ sample pumped by the Titanium-Sapphire laser. Introducing the quantum yield which include the probability of the exciton self-trapping and the efficiency of the detection unit, Equation (10) can be written as:

$$
P_{f}=\eta \alpha V I_{o}^{3}
$$

In our experimental setting described before, the focal spot are about $1590 \mu \mathrm{m}^{2}$ and the $\mathrm{ZnO}$ film has $2 \mu \mathrm{m}$ thickness, thus the illuminated volume is about $3180 \mu \mathrm{m}^{3}$ and $\eta$ was estimated to by $10^{-5}$ in our experimental set up. The value of $(a)$ is found from the measurements of $P_{f}$ at different values of $I_{o}$, and by applying Equation (11), it was found to by about of $0.0142 \mathrm{~cm}^{3} / \mathrm{GWatt}^{2}$. The result which is not far away from the calculate result from Gaussian fit technique as follow:

The normalized energy transmittance for 3PA of the open aperture z-scan is given by R. L. Sutherland [27]:

$$
\begin{aligned}
T(z)= & \frac{1}{\pi^{1 / 2} p_{o}} \\
& \times \int_{-\infty}^{\infty} \ln \left\{\left[1+p_{o}^{2} \exp \left(-2 x^{2}\right)\right]^{1 / 2}+p_{o} \exp \left(-x^{2}\right)\right\} \mathrm{d} x
\end{aligned}
$$

where $P_{o}=\left(2 \gamma I_{o} L_{e f f}^{\prime}\right)^{1 / 2}, \quad I_{o}=I_{o o} /\left(1+z^{2} / z_{o}^{2}\right)$ is the excitation intensity at the position $z, \quad z_{o}=\pi \omega_{o}^{2} / \lambda$ where $z_{o}$ is the Rayleigh range, $\omega_{o}$ is the minimum beam waist at focal point $(z=0), \lambda$ is the laser free-space wavelength, $L_{\text {eff }}^{\prime}=\left[1-\exp \left(-2 a_{o} L\right)\right] / 2 a_{o}$ is the effective sample length for 3PA processes; $L$ is the sample length and $a_{o}$ is the linear absorption coefficient. The open aperture z-scan graph is always normalized to linear transmittance i.e., transmittance at large values of $|z|$. If $P_{o}<1$ Equation (12) can be expanded in a Taylor series as:

$$
T=\sum_{m=1}^{\infty}(-1)^{m-1} \frac{p_{o}^{2 m-2}}{(2 m-1) !(2 m-1)^{1 / 2}}
$$

Furthermore, if the higher order terms are ignored, the transmission as a function of the incident intensity is given by R. L. Sutherland [27]:

$$
T=1-\frac{\gamma I_{o}^{2} L_{e f f}^{\prime}}{3^{3 / 2}}
$$

The 3PA coefficient can be extracted from the best fit for Equation (14). The sold curve in Figure 12 is the best fit for Equation (14). The Equation (14) shows clearly that the depth of the absorption dip is linearly proportional to the 3PA coefficient $\gamma$, but the shape of the trace is primarily determined by the Rayleigh range of the focused Gaussian beam. The fitted value of $\gamma$ is on the order of $0.0166 \mathrm{~cm}^{3} / \mathrm{Gwatt}^{2}$. This value is five times of magnitudes higher than the value observed with bulk $\mathrm{ZnO}$ sample. The natural logarithm of the $(1-T)$ values are plotted as a function of natural logarithm of the incident intensity $I_{o}$ in Figure 13. The curve can be reasonably fitted with a straight line with slope of 1.99 , this indicate that the 3PA was occur in $\mathrm{ZnO}$ pump by $800 \mathrm{~nm}$ laser source of $60 \mathrm{fs}$ pulse duration. 


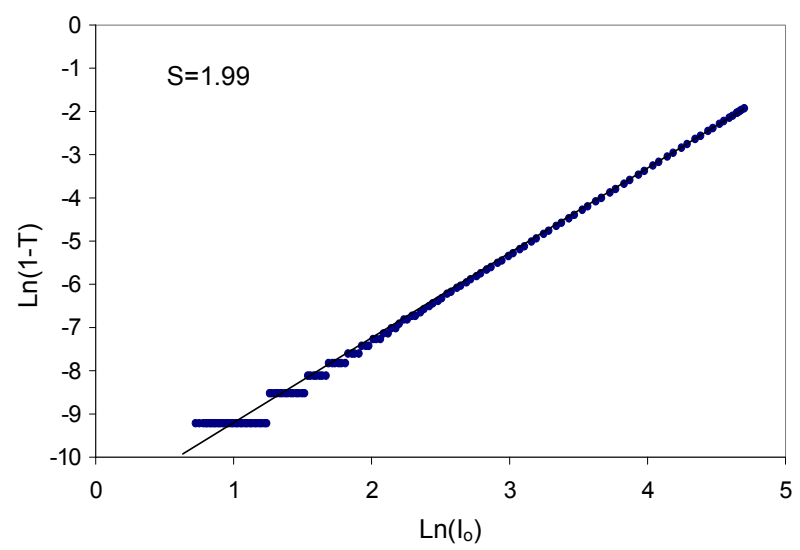

Figure 13. Plot of $\operatorname{Ln}(1-T)$ versus $\operatorname{Ln}\left(I_{o}\right)$ at $800 \mathrm{~nm}$ wavelength, the solid line is the example of the linear at $800 \mathrm{~nm}$ with slope $s=1.99$.

\section{Conclusion}

The three photon absorption has been observed in $\mathrm{ZnO}$ nanocrystalline prepared by chemical method upon illuminating it by femtosecond Titanium-Sapphire laser. The fully computerized $z$-scan system was used to measure the nonlinear absorption coefficient of the prepared samples. The value of the measured nonlinear coefficient was found to be five times higher than the bulk value. The enhancement of the nonlinear coefficient was attributed to the formation of the nanocrystallites of $\mathrm{ZnO}$ and to the existence of the exciton in the prepared film.

\section{Acknowledgements}

This work has been carried out in the physics Department, School of Engineering and Applied Sciences, Harvard University. The authors would like to thanks Mazur Research Group in Harvard University for their help through this work. Thank also to Christopher C. Evans, Jonthan D. B. Bradley, and Eric Mazur for their interest, guide and useful discussion. We thanks also the Ministry of Higher Education in the republic of Iraq for support this work.

\section{REFERENCES}

[1] Y. W. Zhu, et al., "Multiwalled Carbon Nanotubes Beaded with $\mathrm{ZnO}$ nanoparticles for Ultrafast Nonlinear Optical Switching," Advanced Materials, Vol. 18, No. 5, 2006, pp. 587-592. doi:10.1002/adma.200501918

[2] K. Ramanathan, J. Keane and R. Noufi, "Properties of High-Efficiency CIGS Thin-Film Solar Cells," NREL/CP, Vol. 520, 2005, Article ID: 37404.

[3] C. L. Rhodes, S. Lappi, D. Fischer, S. Sambasivan, J. Genzer and S. Franzen, "Characterization of Monolayer Formation on Aluminum-Doped ZincOxide Thin Films," American Chemical Society, Vol. 24, No. 2, 2008, pp. 433-440.
[4] H. Li, et al., "Microstructural Study of MBE-Grown $\mathrm{ZnO}$ Film on GaN/Sapphire (0001) Substrate," Central European Journal of Physics, Vol. 6, No. 3, 2008, pp. 638-642. doi:10.2478/s11534-008-0032-2

[5] T. Sato, et al., "Production of Transition Metal-Doped ZnO Nanoparticles by Using RF Plasma Field," Journal of Crystal Growth, Vol. 275, No. 1-2, 2005, pp. 983-987. doi:10.1016/j.jcrysgro.2004.11.152

[6] S. Singh and M. S. R. Rao, "Structure and Physical Properties of Undoped $\mathrm{ZnO}$ and Vanadium Doped $\mathrm{ZnO}$ Films Deposited by Pulsed Laser Deposition," Journal Nanoscience and Nanotechnology, Vol. 8, No. 5, 2007, pp. 2575-2577.

[7] Ü. Özgür, et al., "A Comprehensive Review of ZnO Materials and Devices," Journal of Applied Physics, Vol. 98, No. 4, 2005, Article ID: 041301. doi:10.1063/1.1992666

[8] J.-H. Lin, et al., "Two-Photon Resonance Assisted Huge Nonlinear Refraction and Absorption in ZnO Thin Films Institute of Electro-Optical Engineering," Journal of Applied Physics, Vol. 97, No. 3, 2005, Article ID: 033526. doi:10.1063/1.1848192

[9] S. J. Bentley, et al., "Three-Photon Absorption for Nanosecond Excitation in Cadmium Selenide Quantum Dots," Optical Engineering, Vol. 46, No. 12, 2007, Article ID: 128003. doi: $10.1117 / 1.2823156$

[10] E. W. Van Stryiand, M. Sheik-Bahae, A. A. Said and D. J. Hagan, "Characterization of Nonlinear Optical Absorption and Refraction," Progress in Crystal Growth and Characterization of Materials, Vol. 27, No. 3-4, 1993, pp. 279-311. doi:10.1016/0960-8974(93)90026-Z

[11] J. He, W. Ji and J. Mi, "Three-Photon Absorption in Water-Soluble ZnS Nanocrystal," Applied Physics Letters, Vol. 88, No. 18, 2006, Article ID: 181114. doi:10.1063/1.2198823

[12] B. Gu, et al., "Three-Photon Absorption Saturation in ZnO and ZnS Crystals," Journal of Applied Physics, Vol. 103, No. 7, 2008, Article ID: 073105. doi:10.1063/1.2903576

[13] S. Pearl, et al., "Three Photon Absorption in Silicon for 2300 - 3300 nm," Applied Physics Letters, Vol. 93, No. 13, 2008, Article ID: 131102. doi:10.1063/1.2991446

[14] A. Penzkofer and W. Falkenstein, "Three Photon Absorption and Subsequent Excited-State Absorption in CdS," Optics Communications, Vol. 16, 1976, pp. 247-250.

[15] M. G. Vivas, T. Shih, T. Voss, E. Mazur and C. R. Mendonca, "Nonlinear Spectra of ZnO: Reverse Saturable, Two- and Three-Photon Absorption," Optics Express, Vol. 18, No. 9, 2010, pp. 9628-9633. doi:10.1364/OE.18.009628

[16] A M. Suhail, H. J. Kbashi and R. K. Jamal, “Three-Photon Absorption in Nanostructure Wide-Band Gap Semiconductor ZnO Using Femtosecond Laser," Modern Applied Science, Vol. 5, No. 6, 2011, pp. 199-210. doi:10.5539/mas.v5n6p199

[17] M. R. Islam and J. Podde, "Optical Properties of $\mathrm{ZnO}$ Nanofiber Thin Film Grown by Spray Pyrolysis of Zinc Acetate Precursor," Crystal Research and Technology, Vol. 44, No. 3, 2009, pp. 286-292. 
doi: $10.1002 /$ crat.200800326

[18] A. L. Patterson, "The Scherrer Formula for X-Ray Particle Size Determination," Physical Reviews, Vol. 56, No. 10, 1939, pp. 978-982. doi:10.1103/PhysRev.56.978

[19] K. Yim and C. Lee, "Optical Properties of Al-Doped ZnO Thin Films Deposited by Two Different Sputtering Methods," Crystal Research and Technology, Vol. 41, No. 12, 2006, pp. 1198-1202. doi:10.1002/crat.200610749

[20] C. X. Xu, et al., "Growth and Spectral Analysis of $\mathrm{ZnO}$ Nanotubes," Journal of Applied Physics, Vol. 103, No. 9, 2006, Article ID: 094303. doi:10.1063/1.2908189

[21] D. D. Wang, J. H. Yang, L. L. Yang, Y. J. Zhang, J. H. Lang and M. Gao, "Morphology and Photoluminescence Properties of ZnO Nanostructures Fabricated with Different Given Time of Ar," Crystal Research and Technology, Vol. 43, No. 10, 2008, pp. 1041-1045. doi:10.1002/crat.200800109

[22] K. Alim, V. A. Fonoberov, M. Shamsa and A. A. Balandin, "Micro-Raman Investigation of Optical Phonons in $\mathrm{ZnO}$ Quantum Dots," Journal of Applied Physics, Vol. 97, No.
12, 2005, Article ID: 124313. doi:10.1063/1.1944222

[23] N. Ashkenov, B. N. Mbenkum, C. Bundesmann, V. Riede, M. Lorenz, D. Spenmann and E. M. Kaidashev, "Infrared Dielectric Functions and Phonon Modes of High-Quality ZnO Film," Journal of Applied Physics, Vol. 93, No. 1, 2003, pp. 126-133. doi:10.1063/1.1526935

[24] H. Richer, Z. P. Wany, "The One Phone Raman Spectrum in Microcrystalline Silicon," Solid State Communications, Vol. 39, No. 5, 1981, pp. 625-629. doi:10.1016/0038-1098(81)90337-9

[25] I. H. Chambell and P. M. Fanchet, "Three Effects of Microcrystal Size and Shape on the One Phonon Raman Spectra of Crystalline Semiconductors," Solid State Communications, Vol. 58, 1986, p. 739.

[26] J. He, et al., "Three-Photon Absorption in $\mathrm{ZnO}$ and $\mathrm{ZnS}$ Crystals," Optics Express, Vol. 13, 2005, pp. 9235-9247.

[27] R. L. Sutherland, D. G. McLean and S. Kirkpatrick, "Handbook of Nonlinear Optics," 2nd Edition, Reserved and Expanded, New York, 2003. 